\title{
Formation Condition of Fiberform Nanostructured Tungsten by Helium Plasma Exposure
}

\author{
Wataru SAKAGUCHI, Shin KAJITA ${ }^{1)}$, Noriyasu OHNO, Makoto TAKAGI \\ and Hiroaki KURISHITA ${ }^{2)}$ \\ Department of Energy Engineering and Science, Graduate School of Engineering, \\ Nagoya University, Nagoya 464-8608, Japan \\ ${ }^{1)}$ EcoTopia Science Institute, Nagoya University, Nagoya 464-8608, Japan \\ 2) International Research Center for Nuclear Materials Science, Institute for Materials Research (IMR), \\ Tohoku University, Oarai, Ibaraki 311-1313, Japan
}

(Received 9 January 2009 / Accepted 16 May 2009)

\begin{abstract}
Tungsten samples were irradiated with high density helium plasma in the divertor plasma simulator NAGDIS to investigate the formation condition of the fiberform nanostructured tungsten. Experimental results indicated that the surface temperature threshold for the formation of nanostructure was approximately $1000 \mathrm{~K}$ when the incident ion energy was $50 \mathrm{eV}$. When the surface temperature was above $1000 \mathrm{~K}$, the width of the nanostructure increased with the surface temperature. Three tungsten samples manufactured by different procedures (ultra fine grained tungsten, ITER-reference tungsten grade and powder metallurgy tungsten) were exposed to the helium plasma. The nanostructure was formed on all samples.
\end{abstract}

(c) 2010 The Japan Society of Plasma Science and Nuclear Fusion Research

Keywords: Helium, Tungsten, Reflectivity, Nanostructure, NAGDIS-II

DOI: $10.1585 /$ pfr.5.S1023

\section{Introduction}

Tungsten is a candidate material for divertor armor in ITER [1] because of its good thermophysical properties, high melting point, low sputtering yield, and a low tritium inventory. Additionally, tungsten is also a candidate material for in-vessel mirror material in fusion devices [2]. However, it has been found that bubbles/holes and fiberform nanostructured tungsten were formed on tungsten surface by irradiating helium plasma [3,4] at the incident ion energy even below the physical sputtering and threshold energy of helium for displacement on tungsten atom, $E_{\mathrm{min}}^{\text {in }}$, which is estimated to be $\sim 790 \mathrm{eV}$ from the following equation:

$$
E_{\mathrm{dis}}=4 M_{1} M_{2} E_{\mathrm{min}}^{\mathrm{in}} /\left(M_{1}+M_{2}\right)^{2}
$$

Here, $M_{1}$ and $M_{2}$ are atomic mass of helium and tungsten, respectively, and $E_{\text {dis }}$ is average displacement energy of tungsten and of $66 \mathrm{eV}$ [5]. This is because thermal vacancies could be dominating trap sites for helium atoms when material temperature is high [3]. When tungsten is used as a material in fusion devices, the nanostructure may cause serious problems because it could lead to the decrease of the optical reflectivity [6] and the thermal conductivity [7]. Moreover, the nanostructure formation enhances the tungsten impurity release from the material in response to the transient heat load accompanied by ELMs (Edge Localized Modes) and disruptions in future fusion device [8]. Therefore, it is necessary to investigate the formation condition and the formation mechanism of the fiberform nanostructure.

In recent studies, it has been revealed that the high helium ion fluence $\left(>10^{25} \mathrm{~m}^{-2}\right.$ ) was necessary to cover the surface with the fiberform nanostructured tungsten $[4,6,9]$. Moreover, the incident helium ion energy was an important parameter to form the nanostructured tungsten. When the incident helium ion energy was above $30 \mathrm{eV}$, the fiberform nanostructure was formed at the surface temperature of $1800 \mathrm{~K}$, although only the helium bubbles and holes were formed when the incident ion energy was $15 \mathrm{eV}$ [6]. Additionally, it was reported that a nanostructured layer thickness of $5 \mu \mathrm{m}$ was observed by irradiating helium plasma at the fluence of $1.1 \times 10^{27} \mathrm{~m}^{-2}$ [9].

In this paper, to investigate the formation condition in detail, helium plasma irradiations are performed at different surface temperatures with a fixed incident helium ion energy. Moreover, helium irradiations are also performed by using tungsten samples manufactured in different procedures. We will discuss the effect of the grain size on the formation process. The helium ion fluence to form the nanostructure is evaluated by the in situ measurement of the optical reflectivity because the optical reflectivity decreases significantly when the surface is covered with the nanostructure. 


\section{Experimental Setup}

Experiments were performed in the divertor plasma simulator NAGDIS-II and NAGDIS-I (NAGoya university DIvertor Simulator). Detailed descriptions of the devices have been reported in $[10,11]$. Figure 1 shows a schematic view of the experimental setup. High density helium plasma, which is produced by dc arc discharge, was irradiated to tungsten samples situated in parallel to the magnetic field line. The optical reflectivity of the sample was measured by using a He-Ne laser, of which wavelength is $632.8 \mathrm{~nm}$, and a photodiode with a band-pass optical filter to exclude the emission of the plasma. A light chopper and a photodiode for reference were installed to remove the background light emission. The sample was polished by sandpapers and alumina suspension before the irradiation. The optical reflectivity before the irradiation was approximately $45 \%$ for the wavelength of $632.8 \mathrm{~nm}$. In order to control the incident ion energy to the samples, the samples were electrically biased in the helium plasma. By considering acceleration in the sheath, the incident angle of helium ion was approximately normal direction to sample surface even though the magnetic field is in parallel to the surface. The surface temperature of the samples was measured by a pyrometer with the emissivity of 0.42 . The incident ion flux was derived by the ion current. After the helium plasma irradiation, the samples were analyzed by a SEM (Hitachi: S-4300) and were measured with a spectrophotometer (Nihon Bunkosya: ARV-47S) for the wavelength from 200 to $900 \mathrm{~nm}$.
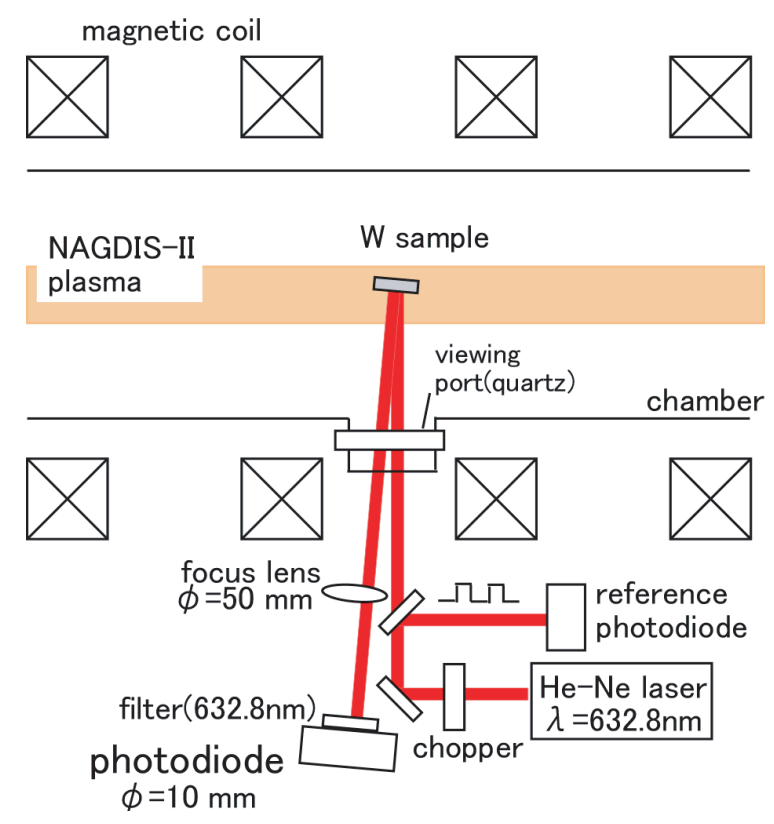

Fig. 1 Schematic illustration of the experimental setup in the divertor simulator NAGDIS-II.

\section{Results and Discussion}

\subsection{Surface temperature dependence}

Figure 2 shows the relative optical reflectivity as a function of helium ion fluence during helium plasma irradiation. Five samples were irradiated with the helium plasma at different surface temperatures from $900 \mathrm{~K}$ to $2040 \mathrm{~K}$. The samples were the powder metallurgy tungsten provided by Nilaco Co. with $99.95 \%$ purity and $0.2 \mathrm{~mm}$ in thickness. The incident helium ion energy was fixed at $50 \mathrm{eV}$. When the surface temperature was $900 \mathrm{~K}$, the relative optical reflectivity decreased to 0.55 at the fluence of $4.5 \times 10^{25} \mathrm{~m}^{-2}$. On the other hand, when the surface temperature was $1130 \mathrm{~K}$, the relative optical reflectivity decreased to $\sim 0$ at the fluence of $3 \times 10^{25} \mathrm{~m}^{-2}$. Similarly, when the surface temperatures were $1400 \mathrm{~K}, 1750 \mathrm{~K}$, and $2040 \mathrm{~K}$, the relative optical reflectivities decreased to $\sim 0$ at the fluence of $5 \times 10^{25} \mathrm{~m}^{-2}, 8 \times 10^{25} \mathrm{~m}^{-2}$, and $8 \times 10^{25} \mathrm{~m}^{-2}$, respectively. When the surface temperature was $900 \mathrm{~K}$, the surface had a metallic luster even after the helium irradiation. When the surface temperature was in the range of $1130-1750 \mathrm{~K}$, the surface color changed to black visually because of the helium irradiation. Figure 3 shows the pictures of sample after helium plasma irradiation at the temperature of (a) $900 \mathrm{~K}$ and (b) $1130 \mathrm{~K}$. When the sur-

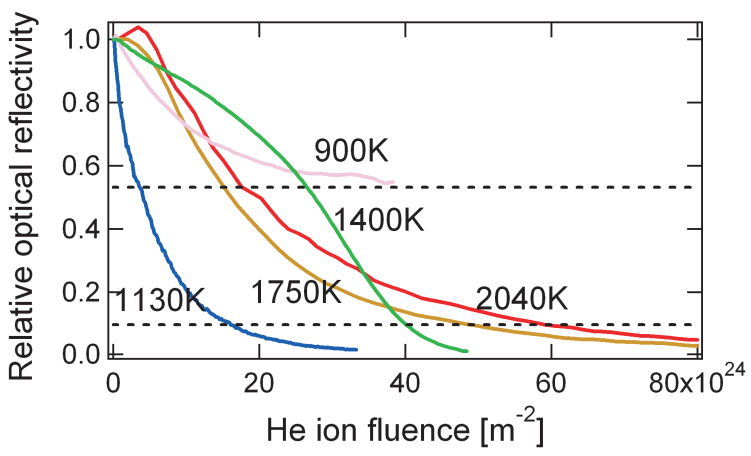

Fig. 2 Measured relative optical reflectivity of tungsten under helium plasma exposure at different sample temperatures from $900 \mathrm{~K}$ to $2040 \mathrm{~K}$ with a fixed incident helium ion energy of $50 \mathrm{eV}$.

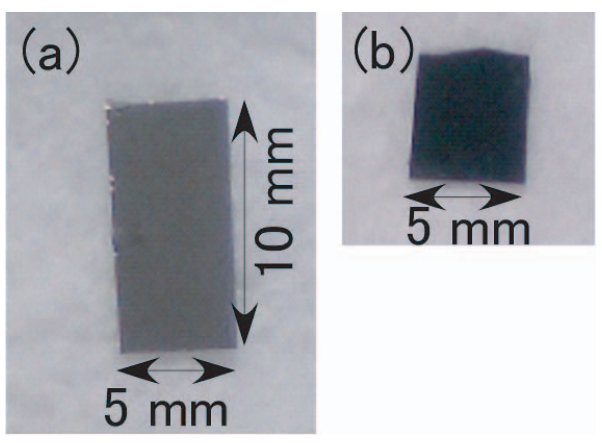

Fig. 3 Pictures of sample after helium plasma irradiation at the temperature of (a) $900 \mathrm{~K}$ and (b) $1130 \mathrm{~K}$. 
face temperature was $2040 \mathrm{~K}$, the surface color changed to silver without metallic luster. The necessary helium ion fluences to decrease the reflectivity to $\sim 0.1$ for $1130 \mathrm{~K}$, $1400 \mathrm{~K}, 1750 \mathrm{~K}, 2040 \mathrm{~K}$ were $1.5 \times 10^{25}, 4 \times 10^{25}, 5 \times 10^{25}$, and $6 \times 10^{25}$, respectively; the necessary helium ion fluence to decrease the reflectivity increased with the temperature in the temperature range of $1130-2040 \mathrm{~K}$.

When the surface temperature was $1400 \mathrm{~K}$, the decreasing rates of the reflectivity before and after the fluence of $2 \times 10^{25} \mathrm{~m}^{-2}$ changed significantly. Although the reason was not well understood, we speculated that the temperature variation during the irradiation might be one of the reasons. The temperature shown in Fig. 2 was the temperature measured with the radiation pyrometer at the time when temperature became stable after the helium irradiation started. However, in a precise sense, the temperature was not stable during the irradiation, and decreased gradually during the helium plasma exposure. When the surface temperature was $1400 \mathrm{~K}$, for example, the temperature decreased by approximately $90 \mathrm{~K}$ during the helium irradiation at the fluence of $5 \times 10^{25} \mathrm{~m}^{-2}$. Note that the temperature was a measurement value with using the emissivity of 0.42 . We suspected that the emissivity might increase because the reflectivity decreased due to the helium irradiation. In the present situation, the temperature was mainly determined by balancing between the incident power from the plasma and the black-body radiation from the sample. The temperature would decrease if the emissivity increased, because the radiation increased with emissivity. Moreover, the decrease of the temperature that was measured at a fixed emissivity was underestimated if the emissivity increased. It was speculated that the formation rate of the nanostructure would be faster and become close to that at $1130 \mathrm{~K}$ because of the decrease in the temperature. It is necessary for a future work to investigate the physical property change during the irradiation.

Figure 4 shows the SEM micrographs of samples after the helium plasma exposure. The surface that was irradiated at $900 \mathrm{~K}$ had a wavy structure as shown in Fig. 4 (a). In ref. [12], in which the helium irradiation has been performed at a similar temperature but a higher incident ion

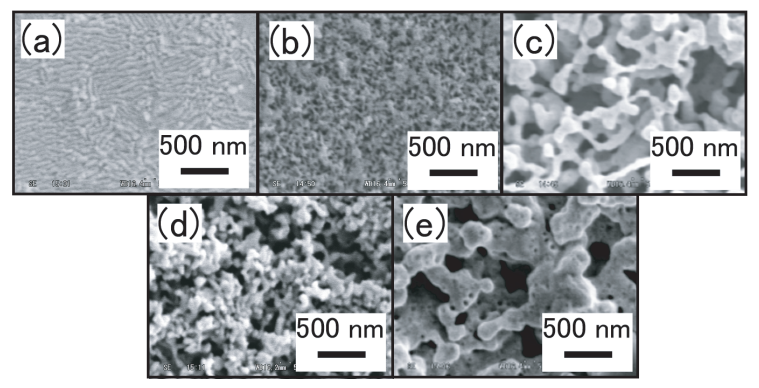

Fig. 4 SEM micrographs of the tungsten surface after the helium plasma exposure. The sample temperatures are (a) $900 \mathrm{~K}$, (b) $1130 \mathrm{~K}$, (c) $1400 \mathrm{~K}$, (d) $1750 \mathrm{~K}$, and (e) $2040 \mathrm{~K}$. energy of $8 \mathrm{keV}$, the reflectivity decreased from $50 \%$ to $20 \%$ at $\lambda=633 \mathrm{~nm}$ for the helium fluence of $3 \times 10^{22} \mathrm{~m}^{-2}$. The decrease in the reflectivity was caused by the surface roughing due to the formation of nanometer sized bubbles. We suspected that small sized bubbles were formed near the surface in Fig. 4 (a), similar to the ion beam irradiation in ref. [12]. The surfaces irradiated at the temperatures of $1130 \mathrm{~K}$ and $1400 \mathrm{~K}$ were covered with the fiberform nanostructure, as shown in Fig. 3 (b) and 3 (c). The results indicated that the sample temperature should be greater than $\sim 1000 \mathrm{~K}$ to form the nanostructure.

Noteworthy is that the width of the structure increased with the temperature. The widths of the structures were, typically, $\sim 20 \mathrm{~nm}$ at $1130 \mathrm{~K}$ (Fig. 4 (b)), $100 \mathrm{~nm}$ at $1400 \mathrm{~K}$ (Fig. 4 (c)), and $\sim 1 \mu \mathrm{m}$ at $2040 \mathrm{~K}$ (Fig. 4 (e)). Although the surface irradiated at $1750 \mathrm{~K}$ had a complicated structure, the width was typically $\sim 200 \mathrm{~nm}$ (Fig. 4 (d)). The formation mechanism of the nanostructure is not entirely understood well. We speculate that the formation of the bubbles and holes is related to the formation of the nanostructure. It has been reported that the sizes of the bubbles increased with the increase of the temperature [3]. In a similar way, the increase of the width of the nanostructure might be related with the growth of the bubble size.

Figure 5 shows the wavelength dependences of reflectivity of the polished sample and samples after the helium plasma exposure. Angle of incident and reflection from normal direction on sample surface was in 7.5 degree. The optical reflectivity at the wavelength of $632.8 \mathrm{~nm}$ corresponds to the one that was measured by using the laser and photodiode. At $\lambda=900 \mathrm{~nm}$, the reflectivity of the sample irradiated at $900 \mathrm{~K}$ decreased by $\sim 20 \%$, while it decreased by $\sim 70 \%$ at $\lambda=200 \mathrm{~nm}$. The degree of reflectivity reduction increases as decreasing the wavelength. From Fig. 4(a), we can see that the size of the surface roughness is approximately several tens $\mathrm{nm}$. The wavelength dependence of the optical reflectivity change could be attributed to the fact that the roughness does not have a significant

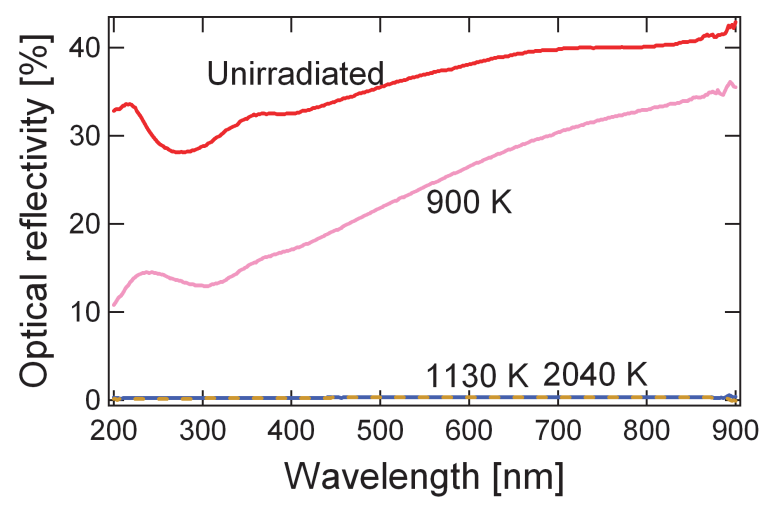

Fig. 5 Reflectivity of the polished sample and the samples that was irradiated to helium plasma at $900 \mathrm{~K}, 1130 \mathrm{~K}$, and $2040 \mathrm{~K}$. 
influence on the optical reflectivity if the wavelength is approximately ten times greater than the size of the structure morphology. When the surface was covered with the fine structure as shown in Fig. 4 (b)-(e), the optical reflectivity became $\sim 0 \%$ in the range of $200<\lambda<900 \mathrm{~nm}$.

The helium fluence at the first mirror in ITER is thought to be on the order of $10^{24} \mathrm{~m}^{-2}$ at maximum for the incident energy of higher than $5 \mathrm{eV}$ [13]. At the helium fluence of $10^{25} \mathrm{~m}^{-2}$, for instance, we can estimate from Fig. 2 that the relative reflectivity would decrease by $\sim 80 \%$ at $1130 \mathrm{~K}$ while it would decrease by $\sim 20 \%$ at $900 \mathrm{~K}$. If the temperature was sufficiently lower than $900 \mathrm{~K}$, the reflectivity would hardly decrease because the nanostructure could not be formed and the formation of bubbles might be also mitigated [3]. For example, when the temperature was $700 \mathrm{~K}$ and the incident energy was $80 \mathrm{eV}$, no morphology change has been observed on the surface at the helium fluence of $1.5 \times 10^{25} \mathrm{~m}^{-2}$, though many nanometersized bubbles were formed in the sub-surface region [14]. The operational temperature of the first mirror in ITER is expected to be $400-500 \mathrm{~K}$; so that it is indicated that the decrease of the reflectivity due to the helium ion irradiation may not be so harmful for in-vessel mirrors when the incident energy is under the displacement energy. However, in actual tokamak situations, the incident energy of the charge-exchanged neutral helium may be higher than the displacement energy, and consequently, knock-on damages may be created even if the material temperature is low. Such radiation damage would act as the effective trapping site of helium atoms for creating the helium bubbles even at the lower dose level $\left(\sim 10^{22} \mathrm{He} / \mathrm{m}^{2}\right)$ [15]. These radiation damages may cause serious problems for in-vessel mirror materials.

\subsection{Different manufacturing procedure}

It has been reported that the grain boundary acted as strong trap sites for helium bubbles [16]. Therefore, we suspected that the grain size might affect the nanostructure formation. Several different tungsten samples were used. They were manufactured in different procedures, so that the grain sizes were different significantly. First one is the ultra fine grained (UFG) W- 0.5 wt. \% TiC consolidates which is fabricated utilizing mechanical alloying in purified Ar and hot isostatic pressing [17]. The average grain size is $\sim 70 \mathrm{~nm}$. Second one is the ITER-reference tungsten grade (ITER-RTG) provided by Plansee Co. The average grain size is $\sim 20 \mu \mathrm{m}$. Third one is the powder metallurgy tungsten (PM-W) provided by Nilaco Co., which was used in Sec. 3.1. The average grain size is few $\mu \mathrm{m}$. Figure 6 shows the relative optical reflectivity as a function of helium ion fluence while helium plasma was irradiated. The incident helium ion energy was $50 \mathrm{eV}$ and the surface temperatures for UFG, ITER-RTG, and PM-W were $1700 \mathrm{~K}$, $1880 \mathrm{~K}$, and $1750 \mathrm{~K}$, respectively. The irradiation condition corresponded to the one that was sufficient to form the

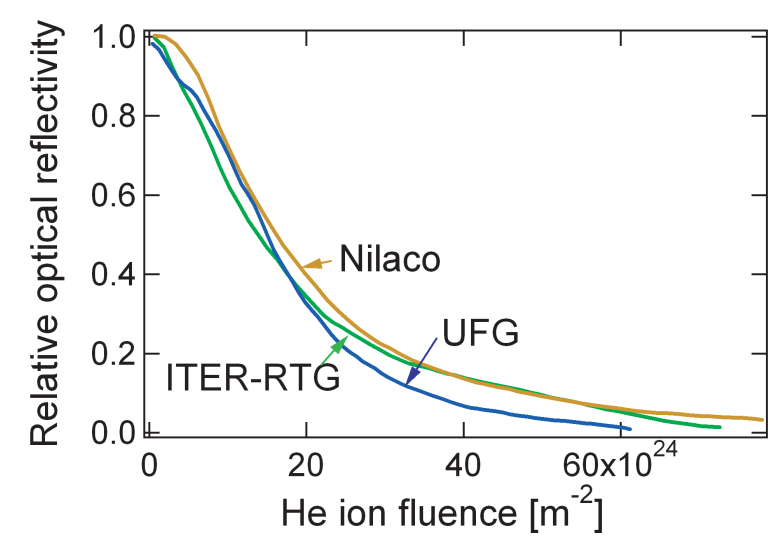

Fig. 6 Measured relative optical reflectivity of tungsten samples that were manufactured by different procedure.

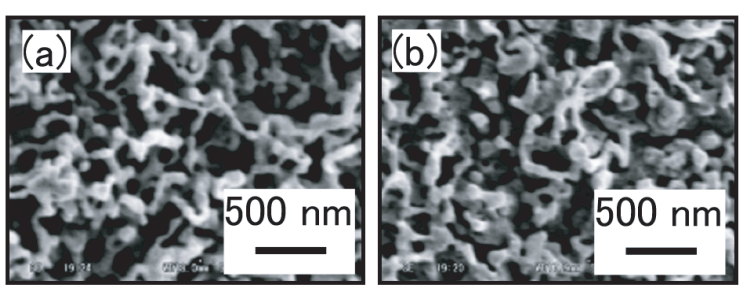

Fig. 7 SEM micrographs of (a) UFG and (b) ITER-RTG after the helium plasma exposure at $\sim 1800 \mathrm{~K}$.
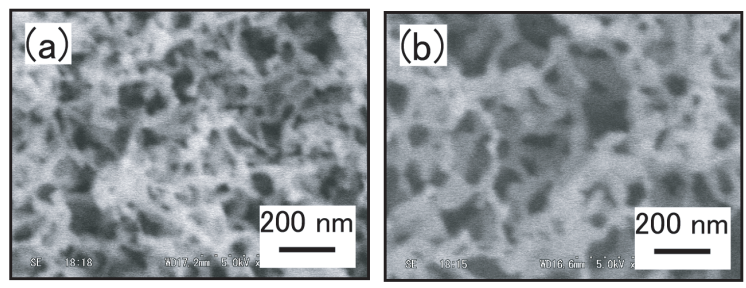

Fig. 8 SEM micrographs of (a) UFG and (b) ITER-RTG after the helium plasma exposure at $\sim 1400 \mathrm{~K}$.

nanostructure on the surface. The helium ion fluence to decrease the reflectivity to $\sim 0$ was almost the same value for the three samples, and was $\sim 7 \times 10^{25} \mathrm{~m}^{-2}$. Figure 7 shows the SEM micrographs of UFG and ITER-RTG after the helium plasma exposure. The widths of the nanostructure were almost same, and were $\sim 100 \mathrm{~nm}$. We performed the irradiation experiments at a lower surface temperature. Figure 8 shows the SEM micrographs of UFG and ITERRTG after the helium plasma exposure at the temperature of $\sim 1400 \mathrm{~K}$. The incident helium ion energy was $50 \mathrm{eV}$. The nanostructure was formed on both samples, and the width of the structure was $\sim 30 \mathrm{~nm}$. The helium ion fluence to form the nanostructure was almost the same value, and was $\sim 6 \times 10^{25} \mathrm{~m}^{-2}$. There was no clear difference on the formation of the nanostructure between the samples with different grain size at the temperature of $\sim 1800 \mathrm{~K}$ and $\sim 1400 \mathrm{~K}$. 
If helium atoms can move only by the diffusion process in the bulk tungsten, the grain size may affect the nanostructure formation. However, experimental result showed that there was no clear difference on the formation of the nanostructure even when the grain size was different. We speculated that the formation of helium hole/bubbles might contribute to the anomalous transport of helium in the bulk tungsten. For example, if pin holes appeared on the surface, helium irradiation would take place through the hole, so that the hole could be a channel to directly transport the helium ions to a deeper region of bulk tungsten. The process might be related with the formation of the nanostructure. However, it is noted that the recrystallization temperature of tungsten has been reported to be in the range of 1423-1973 K [18]; thus the recrystallization might take place during the irradiation. If the recrystallization process would take place, the grain size increased and the originality of the sample was dissipated. It has been discussed in Ref. [18] that the variation of the recrystallization temperature was attributed to the pre-treatment, and the experimental condition. Therefore, we could not distinguish whether the recrystallization took place, particularly at $1400 \mathrm{~K}$. It is expected that irradiation experiments at a significantly lower temperature than the recrystallization one is performed in future.

\section{Conclusion}

Helium ion irradiation experiments were performed to investigate the necessary conditions for the formation of the fiberform nanostructured tungsten. The samples were irradiated with the helium plasma at different surface temperatures with a fixed incident helium ion energy. It was revealed that the necessary minimum sample temperature to form the nanostructure was approximately $1000 \mathrm{~K}$ when the incident ion energy was $50 \mathrm{eV}$. When the surface temperature was lower than $1000 \mathrm{~K}$, the nanostructure was not formed and the reflectivity did not decrease significantly. Moreover, the width of the nanostructure increased with the temperature when the surface temperature was higher than $\sim 1000 \mathrm{~K}$. This result indicates that the formation of nanostructure may be related with the bubble formation, because the bubble size increased with the temperature as well.

Three tungsten samples manufactured by different procedure were exposed to the helium plasma. There were no clear differences on the formation of the nanostructure, even when the grain sizes were different. Although, the results might bring about a clue to understand the formation mechanism of the nanostructure, we could not exclude the possibility that the recrystallization process took place during the irradiation.

For the divertor and in-vessel mirror materials, it is important to prevent the formation of the nanostructure due to the helium irradiation. From the present study, it was revealed that the surface temperature was key factor to the formation of the nanostructure. Therefore, it is expected for the divertor and mirror material that the surface temperature is controlled sufficiently lower than $1000 \mathrm{~K}$.

\section{Acknowledgments}

We wish to thank Dr. Tokitani of NIFS for measuring the optical reflectivity and Dr. Ozawa of Nagoya University for the use of SEM. This research was partially supported by the Ministry of Education, Science, Sports and Culture, Grant-in-Aid for scientific Research (B), 19360413, 2008. This work was also supported in part by Grant-in-Aid for Young Scientists (Start-up), 19860096, from the Japan Society for the Promotion of Science (JSPS).

[1] ITER Physics Basis, Nucl. Fusion 39, 2137 (1999).

[2] A. Litnovsky, V. S. Voitsenya, A. Costley and A. J. H. Donne, Nucl. Fusion 47, 833 (2007).

[3] D. Nishijima, M. Y. Ye, N. Ohno and S. Takamura, J. Nucl. Mater. 329-333, 1029 (2004).

[4] S. Takamura, N. Ohno, D. Nishijima and S. Kajita, PFR 1, 051 (2006).

[5] R. N. Stuart, M. W. Guinan and R. J. Borg, Radiation Effects 30, 129 (1976).

[6] W. Sakaguchi, S. Kajita, N. Ohno and M. Takagi, J. Nucl. Mater. 390-391, 1149 (2009).

[7] S. Kajita, S. Takamura, N. Ohno, D. Nishijima, H. Iwakiri and N. Yoshida, Nucl. Fusion 47, 1358 (2007).

[8] S. Kajita, N. Ohno, W. Sakaguchi and M. Takagi, PFR 4, 004 (2009).

[9] M. J. Baldwin and R. P. Doerner, Nucl. Fusion 48, 035001 (2008).

[10] S. Takamura, N. Ohno and D. Nishijima, Plasma Sources Sci. Technol. 11, A42 (2002).

[11] S. Masuzaki, N. Ohno and S. Takamura, J. Nucl. Mater. 223, 286 (1995).

[12] A. Ebihara, M. Tokitani, K. Tokunaga, T. Fujiwara, A. Sagara and N. Yoshida, J. Nucl. Mater. 363-365, 1195 (2007).

[13] T. Sugie and A. Kukushkin, Private communication.

[14] D. Nishijima, H. Iwakiri, K.Amano, M. Y. Ye, N. Ohno, K. Tokunaga, N. Yoshida and S. Takamura, Nucl. Fusion 45, 669 (2005).

[15] N. Yoshida, H. Iwakiri, K. Tokunaga and T. Baba, J. Nucl. Mater. 337, 946 (2005).

[16] H. Trinkaus and B. N. Singh, J. Nucl. Mater. 323, 229 (2003).

[17] H. Kurishita, S. Matsuo, H. Arakawa, S. Kobayashi, K. Nakai, T. Takida, K. Takabe and M. Kawai, Mater. Sci. Eng. A 477, 162 (2008).

[18] E. Koch-Bienemann, L. Berg and G. Czack, Gmelin Handbook of Inorganic Chemistry (Tungsten) (Springer, Berlin, 1989), Suppl. A3. 